\title{
Perspectives and vision for strain selection in bioaugmentation
}

\author{
Andrew C. Singer, Christopher J. van der Gast and lan P. Thompson
}

Environmental Biotechnology Section, Centre for Ecology and Hydrology - Oxford, Oxford OX1 3SR, UK

\begin{abstract}
Notwithstanding the phenomenally large and everincreasing resource of pollutant-degrading microbial isolates in laboratories around the globe, inoculum survival remains the 'Achilles' heel' for bioaugmentation of contaminated land. Considerable effort has been invested into inoculum strain selection to facilitate pollutant biodegradation, ranging from the isolation of 'superbugs,' which are microorganisms highly resilient to environmental stresses, harboring catabolically superior pollutant-degrading enzymes, to the other extreme in 'priming', where pollutant degradation is carried out through the addition of soil enriched with an undefined consortium of pollutant-degrading microorganisms.
\end{abstract}

The past four years (2001-2004) have seen 172 scientific papers including the keyword 'bioaugmentation' (ISI Web of Science) - nearly double that of the previous four-year period. Nevertheless, this increase has yet to correlate with an increase in the implementation of field-scale bioaugmentation. In this article, we discuss our perspective on the pinch-point for bioaugmentation - strain selection. We suggest that many of the challenges of bioaugmentation can be attacked with greater efficacy through the manipulation of a select subset of the 'superbug' population. We propose that the future of bioaugmentation rests in the coordinated efforts of the international scientific community to harness the unrealized potential of the superbugs, rescuing them from a destiny of serial 'proof of concept' studies. We also highlight two strain-selection techniques that show great promise for further enhancing the efficacy and applicability of bioaugmentation in the field.

Bioaugmentation is an approach rooted in the ancient art of food preparation. Most notably, cheese, yoghurt and beer production employ small quantities of the previous batch of fermented product to initiate the process once again, thereby reproducing with high precision the same product with each successive batch. For millennia, the 'starter culture' for milk fermentation was supplied from the fortuitous storage of cow or goat milk in an animal skin bag. It was not until insights provided by Louis Pasteur in the later half of the 19th century, that dairy science was able to transform from the black-box art form mastered by few, to a six billion dollar (US), scientifically precise industry, mastered by many.

Corresponding author: Singer, A.C. (acsi@ceh.ac.uk).

Available online 24 December 2004
Despite our long cultural history of bioaugmentation, it is only recently that we have attempted scientifically to engineer many of these processes. Bioaugmentation of polluted soil is an approach whereby microorganisms are amended to a contaminated site to hasten the degradation of pollutants. It is through the introduction of excess, active, degrader microorganisms that the pollutant is mineralized, thereby providing remediation at a faster rate than would otherwise occur by means of the indigenous microorganisms. We direct the reader to van Veen et al., for an excellent, comprehensive review of bioaugmentation techniques [1].

Strain selection for bioaugmentation is contingent on our understanding of not only microbial ecology but society. Clearly, recent developments in molecular microbial ecology have greatly assisted our ability to track strains, interrogate environmental systems and appreciate the complexity of soil microbiology in ways that only five years ago were impossible. If a plentiful supply of superbugs are available for exploitation, and our understanding of the target system is increasing rapidly, why are there so few examples of field-scale bioaugmentation? In this paper, we propose that much progress can be gained through a coordinated exploitation of the current knowledge base, molecular biology techniques and global culture collections.

\section{Strain selection}

Strain selection is founded on the principle that certain microorganisms are better suited for particular (catabolic) tasks and environments than others. Traditional bioaugmentation has achieved its greatest results through repeated application of highly competent pollutantdegrading bacteria [2,3]. Arguably, the high frequency of bioaugmentation events is necessary owing to poor survival and activity of the inoculum, which, among a myriad of other reasons, is ultimately founded on strain selection. When designing a bioaugmentation regimen, for obvious reasons, strain selection is confined to those species that are not 'linked' to human pathogens (i.e. share the same genus and species name); however, it is often the case that a 'superbug' is closely related to a human pathogen, precluding its field implementation [4]. This is exemplified by Pseudomonas aeruginosa, a versatile Gram-negative bacterium that grows in soil, marshes and coastal marine habitats and has been utilized as a plant growth-promoting rhizobacterium. Specifically, Pseudomonas aeruginosa strain IE-6 was 
shown to suppress the root-infecting fungi Macrophomina phaseolina, Fusarium oxysporum, Fusarium solani and Rhizoctonia solani in tomato plants [5]. However, a range of different strains of $P$. aeruginosa are also known to infect the lungs of cystic fibrosis sufferers, where it causes inflammation and episodes of intense breathing problems which can be potentially fatal [6]. Hence, strain selection must not be based on performance alone.

\section{Heirloom strains}

The search for novel microorganisms with exceptional catabolic or survival abilities is an ongoing pursuit of many laboratories worldwide. This effort has generated many hundreds of thousands of 'ordinary' microorganisms, along with a notable few with exceptional abilities (i.e. superbugs). A subset of the superbug population, denoted as 'Heirloom' microorganisms, are superbugs that are maintained and handed down from generation to generation within a research group - with each successive transfer adding phenotypic and genotypic details and genetic modifications to its meta-dossier. These Heirloom superbugs are particularly attractive for bioaugmentation for several reasons: they are usually easily cultured, fast growing, thoroughly characterized and accessible worldwide through one of the many culture collections (e.g. American Type Culture Collection (ATCC), Belgian Co-ordinated Collections of Microorganisms (BCCM), Deutsche Sammlung von Mikroorganismen und Zellkulturen GmbH (DSMZ) and Czech Collection of Microorganisms $(\mathrm{CCM})$ ).

Arguably, one of the most exemplary Heirloom microorganisms is Deinococcus radiodurans. This bacterium has been under increasing scientific scrutiny for nearly five decades, accruing a thick phenotypic [7] and genotypic dossier [8]. The bacterium touts a dizzying array of talents, ranging from its natural resistance to genotoxic chemicals, oxidative damage, high levels of ionizing and ultraviolet radiation and dehydration to its genetically engineered acquisitions of tolerance to high temperatures, ability to biodegrade toluene and detoxify a range of potentially hazardous metals [e.g. mercury (II), iron (III), uranium (VI) and chromium (VI)] [9,10]. Ultimately, $D$. radiodurans is being groomed for one of the most difficult of pollutant challenges - bioaugmentation into high-temperature radioactive mixed wastes (i.e. organic and metal pollutants). Despite its awesome potential to remediate among the most challenging and hazardous polluted sites, it is not likely to be readily adopted by problem holders worldwide because of its genetic modifications and unknown impact on the environment. Hence, this superbug could very well find itself in Heirloom purgatory, where it might remain indefinitely, forever stymieing the interests of researchers, funding bodies and problem holders [11,12].

One solution to this problem, in principle supported by Barac et al. [13] and Blumenroth and Wagner-Dobler [14], is that the scientific community coordinates its research efforts on Heirloom microorganisms. The pool of Heirloom species would be chosen based on their amenability to manipulation, exceptional catabolic ability, current knowledge base, tolerance to environmental stresses, technical ease of use and lack of public health threat (e.g. Pseudomonas sp. strain ADP, Pseudomonas sp. NCIB 9816-4, Burkholderia xenovorans LB400, Phanerochaete chrysosporium strain RP78, Methylococcus capsulatus (Bath), Geobacter sulfurreducens, Deinococcus radiodurans). The Heirloom collection would constitute a dynamic pool of superbugs, thoroughly tested for their suitability for field use as well as optimized for enhanced survival and activity in the environment through (genetic) manipulation. The proposal would establish the microbial equivalent of open-source coding (e.g. LINUX), focusing the international research effort into providing a toolbox of workhorse microorganisms upon which all field releases could be carried out, affording the greatest likelihood of achieving the desired objectives while providing the best opportunity to address the safety concerns of the public. In the end, the approach would impart greater value for money to the research-funding body, provide the problem holder with a scientifically sound, costeffective technology to remediate polluted sites and ultimately inspire creative and innovative science, in large part owing to a priori knowledge that the finished product stands a reasonable chance of contributing to the remediation of contaminated land.

\section{In rhizo-directed strain selection}

In a majority of cases, superbugs are isolated through a standard procedure of batch-enrichment culture, which relies on the ability of the isolate to grow rapidly on a specific carbon source in defined chemical and environmental laboratory conditions. Although highly effective in generating superbugs, the approach operates in a vacuum from the natural environment. In an effort to advance the techniques of strain selection beyond traditional batch enrichment, researchers have turned to a relatively new approach, which incorporates the benefit(s) of plants as witnessed in phytoremediation with optimized biodegradative strains [15], called in rhizo-directed strain selection. The approach utilizes a microorganism with the capacity to grow on one or more root exudates within the rhizosphere in addition to degrading the relevant pollutant. The microorganisms, once inoculated into the appropriate plant rhizosphere, would express the pollutant-degrading enzymes, thereby decontaminating the soil while growing on the plant root exudates to sustain its population and metabolic activity. An added benefit of including plants in the bioaugmentation approach is the supply of additional secondary metabolites and root exudates, which might also fortuitously stimulate and maintain the induction of pollutant-degrading enzymes in situ [16,17]. The inoculum, in return, can provide protection to the plant against phytotoxic chemicals and plant pathogens. The synergistic relationship between the plant and the inoculum established by this approach is arguably more resilient, sustainable and cost-effective than the standard bioaugmentation approach. Reliance of the inoculum on plant exudates for survival precludes its unintended dispersal to nontarget soils, thereby providing a mechanism for addressing public safety concerns.

Kuiper et al. [18] were the first to methodically select a strain indigenous to a host plant's rhizosphere that 
exhibited preferential growth on the plant root exudates, good survival in the rhizosphere and enhanced pollutantdegrading ability. To a similar end, Narasimhan et al. [19] engineered a bacterium to utilize the predominant root exudates of Arabidopsis for the remediation of polychlorinated biphenyl (PCB) in soil. This nutritional bias for the inoculum provides a selective advantage for the strain while in the Arabidopsis rhizosphere. The inoculum, a PCB-degrading Pseudomonas putida PML2, was then capable of degrading PCBs upon augmentation into the Arabidopsis rhizosphere, while growing on phenylpropanoids, the main root exudates, as a 'preferential' growth substrate [14]. This study highlights one of the most promising approaches for the future of bioaugmentation: the combination of a sustainable, semiselective nutrient source in plant exudates with the rhizosphere competence of a pollutant-degrading 'superbug' [17].

Additional techniques can be employed in situ to achieve superior strains for bioaugmentation. de Weert et al. [20] demonstrated a novel method of enhancing competitive root-tip colonization in a Pseudomonas fluorescens strain through repeated cycling of the strain and mutant derivatives on the plant root in situ. The authors observed a 100- to 1000-fold increase in the strain's roottip-colonizing ability in tomato and grass plants. The authors concluded that enhanced competitive root-tip colonization was a result of the accumulation of nonlethal mutations, which enabled the strain to rapidly adapt to the plant rhizosphere. This approach to strain selection is particularly attractive because it is rapid, inexpensive, does not require genetic engineering and is readily applicable to Heirloom strains.

\section{Non-traditional strain selection}

Up to this point, we have provided our vision for the future of bioaugmentation: Heirloom species and in rhizodirected strain selection. However, we recognize that significant potential rests in a less traditional approach, termed 'priming'. Priming is generally described as predisposing an isolate or population of microorganisms to future conditions in which they are designed to perform a function. Priming is routinely used in probiotic yogurt culture preparation, where Lactococcus or Bifidobacterium cultures are subjected to the mildly acidic environment of the yogurt, 'priming' the inoculum for the harsh, acidic conditions within the stomach $[21,22]$. This pretreatment, albeit unnecessary for acid-tolerant strains, is essential for the viable transit of nonacid-tolerant strains through the stomach into the intestines [21].

The priming approach has also been effectively demonstrated in environmental systems, whereby a portion of clean soil is enriched for pollutant-degrading microorganisms by repeated biostimulation with the relevant pollu$\operatorname{tant}(\mathrm{s})$. The resulting soil itself, with its highly competent degrader microbial community, is then used as the inoculum for the target polluted soil [23]. This approach has several advantages: (i) the inoculum is a consortium of indigenous microorganisms which is potentially more resilient to stress than a single isolate; (ii) the 'primed' consortium is maintained within its native soil, potentially enhancing its survival in the target soil; and (iii) inclusion of unculturable microorganisms, which might contain one or more highly competent pollutant degraders. In addition, the approach is theoretically expandable to solving the issue of co-contaminated soil (i.e. one with more than one pollutant), which might necessitate the combination of singly primed soils or one soil primed to degrade multiple contaminants.

Takeuchi et al. [24] were among the first to apply priming to contaminated groundwater. The authors initially demonstrated cometabolism of trichloroethylene (TCE) by methanotrophs from a borehole in a contaminated aquifer. They subsequently amended a poorly performing borehole with groundwater from the TCEdegrading borehole, resulting in a rapid decline in the TCE groundwater concentration. Treatment success was attributed to the activity of the bioaugmented indigenous methanotrophs within the 'primed' TCE-degrading groundwater. Albeit a return to the 'black box' method of remediation, instinctively avoided by scientists, this approach is attractive from a practical perspective; it is technically accessible, easily implemented, inexpensive and environmentally friendly. It remains to be seen how effective different matrices respond to priming (e.g. clayprimed soil augmented into sandy target soil, or high $\mathrm{pH}$ primed soil augmented into netural or low $\mathrm{pH}$ target soil). Moreover, the approach has not been suitably tested for potential scale-up to address large contaminated sites; however, priming clearly warrants a more thorough investigation to answer these and other practical questions.

\section{Conclusion}

In time, it is hoped that bioaugmentation can fulfill its potential to remediate contaminated soils and water, in situ. Many of the limitations to its applicability in the field are the result of an uncoordinated effort to address the social concerns of bioremediation along with the scientific interests. To this end, we propose that exploitation of Heirloom species should be coordinated between research laboratories, providing both scientists and regulators with a predictable, (globally) tested selection of microorganisms, which would facilitate pollutant remediation as well as its public acceptance. In addition, we highlight two non-traditional approaches to strain selection, termed in rhizo-directed strain selection and priming, which demonstrate great promise for remediation of polluted matrices but remain largely on the periphery of the discipline.

\section{Acknowledgements}

We wish to thank the Referees for their valuable feedback and suggestions in preparing this manuscript, as well as Stephanie Hunter and Penny Carter for their assistance.

\section{References}

1 van Veen, J.A. et al. (1997) Fate and activity of microorganisms introduced into soil. Microbiol. Mol. Biol. Rev. 61, 121-135

2 Gilbert, E.S. and Crowley, D.E. (1998) Repeated application of carvone-induced bacteria to enhance biodegradation of polychlorinated biphenyls in soil. Appl. Microbiol. Biotechnol. 50, 489-494 
3 Singer, A.C. et al. (2000) Bioremediation of polychlorinated biphenylcontaminated soil using carvone and surfactant-grown bacteria. Appl. Microbiol. Biotechnol. 54, 838-843

4 Rossello-Mora, R. (2003) Opinion: The species problem, can we achieve a universal concept? Syst. Appl. Microbiol. 26, 323-326

5 Siddiqui, I.A. and Shaukat, S.S. (2003) Combination of Pseudomonas aeruginosa and Pochonia chlamydosporia for control of root-infecting fungi in tomato. J. Phytopathol. 151, 215-222

6 Aaron, S.D. et al. (2004) Adult cystic fibrosis exacerbations and new strains of Pseudomonas aeruginosa. Am. J. Respir. Crit. Care Med. $169,811-815$

7 Venkateswaran, A. et al . (2000) Physiologic determinants of radiation resistance in Deinococcus radiodurans. Appl. Environ. Microbiol. 66, 2620-2626

8 White, O. et al. (1999) Genome sequence of the radioresistant bacterium Deinococcus radiodurans R1. Science 286, 1571-1577

9 Brim, H. et al. (2003) Engineering Deinococcus geothermalis for bioremediation of high-temperature radioactive waste environments. Appl. Environ. Microbiol. 69, 4575-4582

10 Lange, C.C. et al. (1998) Engineering a recombinant Deinococcus radiodurans for organopollutant degradation in radioactive mixed waste environments. Nat. Biotechnol. 16, 929-933

11 Arntzen, C.J. et al. (2003) GM crops: science, politics and communication. Nat. Rev. Genet. 4, 839-843

12 Morrissey, J.P. et al. (2002) Exploitation of genetically modified inoculants for industrial ecology applications. Antonie Van Leeuwenhoek 81, 599-606

13 Barac, T. et al. (2004) Engineered endophytic bacteria improve phytoremediation of water-soluble, volatile, organic pollutants. Nat. Biotechnol. 22, 583-588
14 Blumenroth, P. and Wagner-Dobler, I. (1998) Survival of inoculants in polluted sediments: effect of strain origin and carbon source competition. Microb. Ecol. 35, 279-288

15 Kuiper, I. et al. (2004) Rhizoremediation: a beneficial plant-microbe interaction. Mol. Plant Microbe Interact. 17, 6-15

16 Singer, A.C. et al. (2004) The tritrophic trinity: a source of pollutantdegrading enzymes and its implications for phytoremediation. Curr. Opin. Microbiol. 7, 239-244

17 Singer, A.C. et al. (2003) Secondary plant metabolites in phytoremediation and biotransformation. Trends Biotechnol. 21, 123-130

18 Kuiper, I. et al. (2001) Selection of a plant-bacterium pair as a novel tool for rhizostimulation of polycyclic aromatic hydrocarbon-degrading bacteria. Mol. Plant Microbe Interact. 14, 1197-1205

19 Narasimhan, K. et al. (2003) Enhancement of plant-microbe interactions using a rhizosphere metabolomics-driven approach and its application in the removal of polychlorinated biphenyls. Plant Physiol. 132, 146-153

20 de Weert, S. et al. (2004) Generation of enhanced competitive root-tipcolonizing pseudomonas bacteria through accelerated evolution. J. Bacteriol. 186, 3153-3159

21 O'Sullivan, D.J. (2001) Screening of intestinal microflora for effective probiotic bacteria. J. Agric. Food Chem. 49, 1751-1760

22 Duwat, P. et al. (2000) Lactococcus lactis, a bacterial model for stress responses and survival. Int. J. Food Microbiol. 55, 83-86

23 Gentry, T.J. et al. (2004) Functional establishment of introduced chlorobenzoate degraders following bioaugmentation with newly activated soil. Biodegradation 15, 67-75

24 Takeuchi, M. et al. (2004) Natural groundwater of a gas field utilizable for a bioremediation of trichloroethylene-contamination. Environ. Geol. 45, 891-898

\section{Important information for personal subscribers}

Do you hold a personal subscription to a Trends journal? As you know, your personal print subscription includes free online access, previously accessed via BioMedNet. From now on, access to the full-text of your journal will be powered by Science Direct and will provide you with unparalleled reliability and functionality. Access will continue to be free; the change will not in any way affect the overall cost of your subscription or your entitlements.

The new online access site offers the convenience and flexibility of managing your journal subscription directly from one place. You will be able to access full-text articles, search, browse, set up an alert or renew your subscription all from one page.

In order to protect your privacy, we will not be automating the transfer of your personal data to the new site. Instead, we will be asking you to visit the site and register directly to claim your online access. This is one-time only and will only take you a few minutes.

Your new free online access offers you:

- Quick search • Basic and advanced search form • Search within search results • Save search • Articles in press • Export citations - E-mail article to a friend • Flexible citation display • Multimedia components • Help files

- Issue alerts \& search alerts for your journal

http://www.trends.com/claim_online_access.htm 